\title{
Effect of tunnel injection through the Schottky gate on the static and noise behavior of GalnAs/AllnAs high electron mobility transistor
}

\author{
Diego Moro-Melgar, ${ }^{1, a)}$ Javier Mateos, ${ }^{2, b)}$ Tomás González, ${ }^{2, c)}$ and Beatriz G. Vasallo,d) \\ ${ }^{1}$ Observatoire de Paris, LERMA, 77 avenue Denfert Rochereau 75014 Paris, France \\ ${ }^{2}$ Departamento de Física Aplicada, Universidad de Salamanca, Plaza de la Merced s/n, 37008 Salamanca, \\ Spain
}

(Received 14 June 2014; accepted 28 November 2014; published online 15 December 2014)

\begin{abstract}
By using a Monte Carlo simulator, the influence of the tunnel injection through the Schottky contact at the gate electrode of a GaInAs/AlInAs High Electron Mobility Transistor (HEMT) has been studied in terms of the static and noise performance. The method used to characterize the quantum tunnel current has been the Wentzel-Kramers-Brillouin (WKB) approach. The possibility of taking into account the influence of the image charge effect in the potential barrier height has been included as well. Regarding the static behavior, tunnel injection leads to a decrease in the drain current $\boldsymbol{I}_{\boldsymbol{D}}$ due to an enhancement of the potential barrier controlling the carrier transport through the channel. However, the pinch-off is degraded due to the tunneling current. Regarding the noise behavior, since the fluctuations in the potential barrier height caused by the tunnelinjected electrons are strongly coupled with the drain current fluctuations, a significant increase in the drain-current noise takes place, even when the tunnel effect is hardly noticeable in the static $I-V$ characteristics, fact that must be taken into account when designing scaled HEMT for low-noise applications. In addition, tunnel injection leads to the appearance of full shot noise in the gate current. (C) 2014 AIP Publishing LLC. [http://dx.doi.org/10.1063/1.4903971]
\end{abstract}

\section{INTRODUCTION}

InP-based HEMTs have demonstrated exceptional properties for high frequency applications due to their high cutoff frequencies as well as their excellent noise performance. ${ }^{1-3}$ Traditionally, to further improve the frequency operation of these devices, the gate length $L_{g}$ has been reduced down to the technological limit. However, in order to avoid the socalled short-channel effects, a vertical scaling of the layer structure must go along with the reduction of $L_{g}$ in order to keep a high aspect ratio (ratio between $L_{g}$ and the distance between the gate electrode and the 2-D channel electron gas, a), which is considered as the fundamental restriction of HEMTs. $^{4}$ In fact, this scaling rule is limited by the emergence of a leakage current through the Schottky barrier at the gate electrode, typically when $a$ is reduced to less than $8-10 \mathrm{~nm}$. The aim of this paper is to perform a deep study of the physical consequences of the appearance of direct tunnel injection through the Schottky barrier at the gate-contact in GaInAs/AlInAs HEMTs, in terms of their static behavior and noise performance. To this purpose, we make use of a semiclassical two-dimensional Monte Carlo (MC) simulator $^{4-6}$ in which the tunnel injection of carriers through the gate electrode has been included by means of the Wentzel-KramersBrillouin (WKB) method. ${ }^{7}$ The WKB approach is able to correctly predict (as compared to other more elaborated but CPU consuming methods such as the Airy transfer matrix) the current through the Schottky barrier by means of an

\footnotetext{
a)E-mail: diego.moro-melgar@obspm.fr.

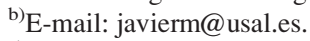

${ }^{c)}$ E-mail: tomasg@usal.es.

${ }^{\mathrm{d})}$ E-mail: bgvasallo@usal.es.
}

adequate consideration of the model parameters (as the Richardson constant, $A$ ). ${ }^{8}$ The $\mathrm{MC}$ model has been proved to be a very helpful tool in the analysis of the noise behavior in devices in which an accurate description of the microscopic dynamics is essential. In this case, we will analyze the noise associated to the electrons injected through the gate.

This work is organized as follows. In Sec. II, the structure under analysis and the physical model employed for the tunnel injection through the Schottky barrier of the gate electrode are detailed. The main results concerning the influence of the tunnel current on the static and noise behavior of an InP-based HEMT are presented in Sec. III. The study will be developed in a standard $100 \mathrm{~nm}$-gate length HEMT (where short channel effects are still weak) in which the tunnel effect has been artificially increased to isolate and clearly evidence its influence, more difficult to identify in smaller HEMTs where leakage current and other short-channel effects can take place. Finally, in Sec. IV, the most important conclusions of this analysis are drawn.

\section{PHYSICAL MODEL}

For calculations, we make use of a semiclassical ensemble MC method self-consistently coupled with a 2D Poisson solver, which includes the influence of degeneracy in the electron accumulation appearing in the channel by using the rejection technique. ${ }^{6}$ The structure under analysis, plotted in Fig. 1, is a $100 \mathrm{~nm}$-gate recessed HEMT, whose epilayer consists of a non-simulated InP substrate, a $200 \mathrm{~nm} \operatorname{In}_{0.52} \mathrm{Al}_{0.48} \mathrm{As}$ buffer, a $20 \mathrm{~nm} \mathrm{In}_{0.53} \mathrm{Ga}_{0.47} \mathrm{As}$ channel, and three $\operatorname{In}_{0.52} \mathrm{Al}_{0.48}$ As layers between the gate-Schottky contact and the channel (a $5 \mathrm{~nm}$ Spacer, a $5 \cdot 10^{12} \mathrm{~cm}^{-2} \delta$-doped layer modeled as a $5 \mathrm{~nm}$ layer doped at $\mathrm{N}_{\mathrm{D}}=10^{19} \mathrm{~cm}^{-3}$ and a $12 \mathrm{~nm}$ Schottky layer), which 


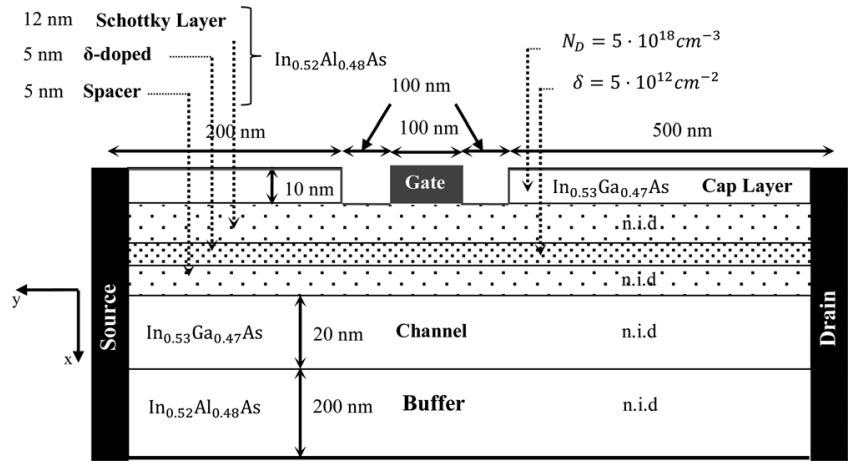

FIG. 1. Topology of the $100 \mathrm{~nm}$-gate-length HEMT under analysis.

will be of key importance when considering the tunnel current through the Schottky contact; and, finally, a cap layer (doped with $\mathrm{N}_{\mathrm{D}}=5 \cdot 10^{18} \mathrm{~cm}^{-3}$ ). For the III-V semiconductor band model, three non-parabolic spherical valleys $(\Gamma, \mathrm{L}$, and $\mathrm{X})$ are considered, and interactions with ionized impurities, alloy, polar and non-polar optical phonons, acoustic phonons and intervalley scattering mechanisms are taken into account. The MC model for InP-based HEMTs in the absence of tunnel injection has been largely validated by means of the agreement between simulation results and experimental measurements related to static, dynamic and noise behavior. ${ }^{9,10}$ The Ramo-Shockley theorem ${ }^{11-13}$ is used in this work for obtaining the instantaneous values of the current in each contact. When tunnel current through the gate is very small, the DC current is calculated by particle counting, which provides a more accurate timeaverage estimation. To properly implement the current injection through the Schottky barrier by means of tunnel and thermionic effect, some specific features have been included in our MC model. Absorption of tunneling carriers from the semiconductor into the metallic region is neglected, since the InAlAs layers remain practically empty of electrons when reverse biasing the gate electrode (furthermore, the channel is sufficiently far from the Schottky contact). In order to consider the injection from the metal into the Schottky layer, we perform a discretization of the incident electrons energy. ${ }^{14-16}$ To illustrate this point, the conduction band in absence of tunnel

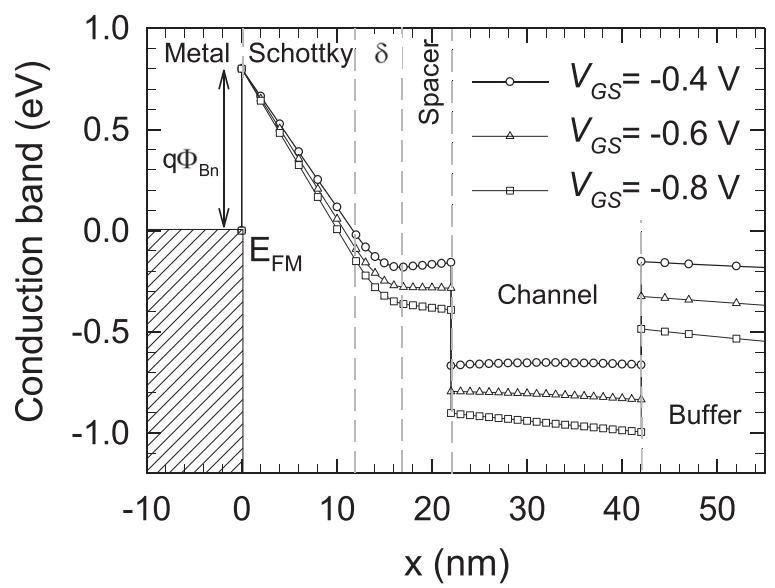

FIG. 2. Conduction band in a cross section in the middle of Schottky contact with $q \phi_{B n}=0.8 \mathrm{eV}, V_{D S}=1.2 \mathrm{~V}$. injection is shown in Fig. 2 along the cross section in the middle of the gate contact, for $V_{D S}=1.2 \mathrm{~V}$ and different $V_{G S}$. In this figure, the Fermi level at the metal $\mathrm{E}_{\mathrm{FM}}$ is taken as the energy origin. A barrier height $\mathrm{q} \phi_{\mathrm{Bn}}=0.8 \mathrm{eV}$ has been considered according to experimental estimations. ${ }^{9,10}$ Two ranges of energies can be distinguished: thermionic energies (related to particles with incident energy higher than $\mathrm{q} \phi_{\mathrm{Bn}}$ ) and tunneling energies (with energy lower than $\mathrm{q} \phi_{\mathrm{Bn}}$ ). In order to implement the tunnel injection through the Schottky barrier it is necessary to calculate the number of injected particles by tunnel effect at each time step in the simulation $(\Delta t=1 \mathrm{fs})$ in the whole energy range. The charge (per unit length in the nonsimulated direction) to be injected in the energy sub-interval $i$ of the cross-sectional subsection $j$ is given by ${ }^{17,18}$

$$
\begin{aligned}
Q_{i}(j)= & \frac{A^{*} T \Delta t}{k_{B}} \cdot \Delta y(j) \cdot T_{c}\left(E_{i}(j)\right) \cdot f_{m}\left(E_{i}(j)\right) \\
& \times\left[1-f_{s c}\left(E_{i}(j)\right)\right] \cdot \Delta E_{i}(j), \\
& i=1, \ldots, N_{s b} ; j=1, \ldots, j_{\max } .
\end{aligned}
$$

To this end, the energy range has been discretized in $N_{s b}$ energy sub-levels and the gate contact in $j_{\max }$ sub-sections in the $Y$ direction; $f_{m}(E)$ and $f_{S C}(E)$ are, respectively, the metal and semiconductor Fermi-Dirac distributions. $E_{i}(j)$ is the energy value corresponding to the energy sub-level $i$ in the cross-sectional subsection $j, \Delta y(j)$ is the transversal width of the cross-sectional subsection $j, \Delta E_{i}(j)=E_{i+1}(j)-E_{i}(j)$ and $T_{c}\left(E_{i}(j)\right)$ is the transmission coefficient through the Schottky barrier for the energy $E_{i}(j) . T$ is the temperature, $k_{B}$ the Boltzmann constant and $A^{*}$ the effective Richardson constant, which can be considered as an adjustable parameter to reproduce experimental results. ${ }^{8,14-16}$ From Eq. (1), the average number of pseudo-particles injected at each time step through the whole gate is

$$
N_{p}=\sum_{j=1}^{j_{\max }} \sum_{i=1}^{N_{s b}} N_{p ; i}(j)=\sum_{j=1}^{j_{\max }} \sum_{i=1}^{N_{s b}} \frac{1}{q} Q_{i}(j) \cdot Z,
$$

where $N_{p: i}(j)$ is the number of pseudo-particles injected at the energy sub-level $i$ in the cross-sectional subsection $j, q$ is the electron charge and $Z$ the non-simulated dimension.

Poissonian statistics is used to include the injection randomness by defining an injection rate $\Gamma=N_{p} / \Delta t$ to determine the time between two consecutive pseudo-particle injections, ${ }^{19}$

$$
t_{i n j}=-\frac{1}{\Gamma} \ln (r),
$$

where $r$ is a random number uniformly distributed between 0 and 1 . Once a specific instant inside the time step $\Delta t$ is selected for a pseudo-particle injection, we determine in a random way the specific energy sub-level $m$ and crosssectional subsection $s$ where the particle will be injected by means of another random number $r^{\prime},{ }^{14}$ as

$$
\sum_{j=1}^{s} \sum_{i=1}^{m} N_{p ; i}(j) \leq r^{\prime} N_{p} \leq \sum_{j=1}^{s} \sum_{i=1}^{m+1} N_{p ; i}(j) .
$$


This expression determines, by knowing $E_{m}(s)$, the $x$ position (corresponding to energy $E_{m}$ in the barrier) inside a subsection $s$ ( $y$ position) where the pseudo-particle is injected. The concrete $y$ position inside the selected sub-section $s$ is determined randomly, considering a uniform probability along the mesh.

Finally, from the energy level selected in the previous step for the injected pseudo-particle, the momentum component parallel to the injection direction is considered to be null $\left(k_{x}=0\right)$ and the thermal energy distribution is used to calculate $k_{y}$ and $k_{z}$.

Regarding the transmission coefficient through the Schottky barrier in Eq. (1), it is calculated following the WKB model. The expression to calculate the transmission coefficient at energy $E, T_{c}(E)$, through a given energy barrier $q V(x)$ is ${ }^{7,17}$

$$
T_{c}(E)=\exp \left[-\frac{2}{\hbar} \int_{x_{1}}^{x_{2}} \sqrt{2 m^{*}[q V(x)-E]} d x\right],
$$

where $x_{1}$ and $x_{2}$ are the classical turning points for $q V(x)$. In our case, $V(x)$ is obtained at each time step from the solution of the Poisson equation in the MC simulation, and the $N_{p}$ and $\Gamma$ are updated accordingly.

Since the WKB transmission probability depends on both the incident energy and the potential profile, the latter can be modified in order to include the effect of the image charges (IC), the so-called Schottky effect. This effect leads to a reduction in the effective barrier height according to the expression $^{23}$

$$
\Delta \phi_{B n}=\sqrt{\frac{q E_{0}}{4 \pi \varepsilon_{S C}}},
$$

where $\varepsilon_{S C}$ is the semiconductor permittivity and $E_{0}$ is the electric field in the contact proximities.

Fig. 3 illustrates the total expected injected charge obtained by the WKB model in the gate electrode of the HEMT in Fig. 1, considering the energy barrier as triangular

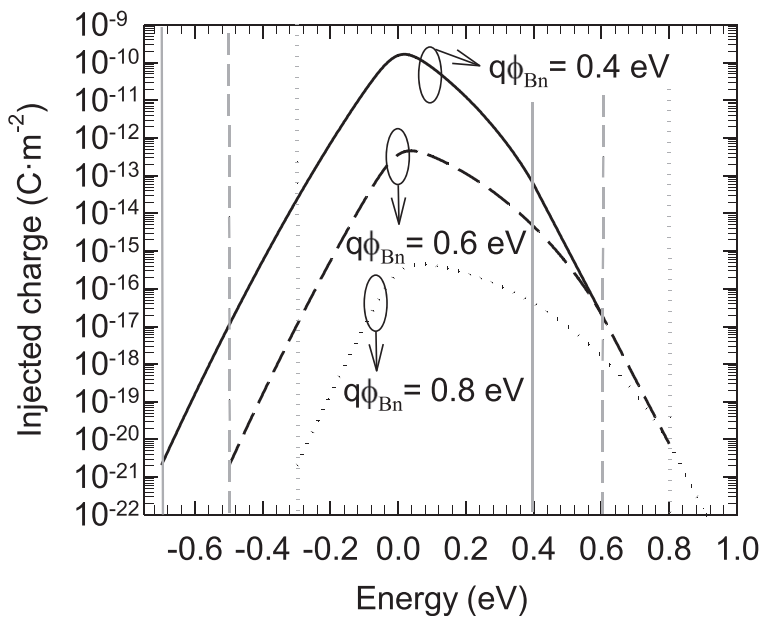

FIG. 3. Injected charge through triangular energy barriers with different energy barrier heights $q \phi_{B n}$. (as presented in Fig. 2) for $V_{G S}=-0.6 \mathrm{~V}$ and different $q \phi_{B n}$, with $A^{*}=10 A \cdot \mathrm{cm}^{-2} \cdot \mathrm{K}^{-2}$ (a standard value for $\operatorname{In}_{0.52} \mathrm{Al}_{0.48} \mathrm{As}$ following ${ }^{20}$ ). The maximum in the tunnel charge injection is found around the metal Fermi level because, despite $T_{c}(E)$ increases with $E, f_{m}(E)$ decreases faster. Then, most of the injected charge is focused at $6 \mathrm{~nm}$ inside the Schottky layer for $q \phi_{B n}=0.4 \mathrm{eV}$ and $10 \mathrm{~nm}$ for $q \phi_{B n}=0.8 \mathrm{eV}$, far away from the channel (Fig. 1). When $E \geq q \phi_{B n}, T_{c}(E)=1$ and the injection is due to thermionic emission. Fig. 3 shows that thermionic emission is negligible compared to tunnel injection. Values of $I_{G}$ as low as the experimental ones(of the order of $0.015 \mathrm{~mA} / \mathrm{mm}$ ) can be found when considering $q \phi_{B n}=0.8 \mathrm{eV}, A^{*}=10 A \cdot \mathrm{cm}^{-2} \cdot \mathrm{K}^{-2}$ and including image charge effect.

In order to clearly predict the influence of the tunnel injection appearing in InGaAs/InAlAs HEMTs when $L_{G}$ (and thus the distance between the Schottky contact and the channel) is reduced, we have analyzed the $100 \mathrm{~nm}-L_{G}$ HEMT in Fig. 1 (thus in the absence of too strong shortchannel effects which could mask the tunnel signature) but with an anomalously high tunneling current. To this aim, values of $A^{*}=10 \mathrm{~A} \cdot \mathrm{cm}^{-2} \cdot \mathrm{K}^{-2}$ and $q \phi_{B n}=0.3 \mathrm{eV}$ will be considered. This value of $q \phi_{B n}$ is sufficiently low so that the tunnel injection is largely noticeable in the static and noise behavior of the device, while the thermionic contribution remains negligible. Even if this may appear to be a limitation of our model, the effects shown in Sec. III, should be qualitatively similar in ultra-scaled HEMTs with very short gate-channel distance and a realistic barrier height, but shortchannel effects could mask the influence of the tunneling current. Additional phenomena such as trap-assisted tunneling, important in GaN HEMTs,${ }^{24}$ could enhance tunneling effects, but here are not considered since, thanks to the advanced technology of InGaAs HEMTs, traps are expected to be absent in the InAlAs Schottky layer.

For the noise analysis, the spectral density of the current fluctuation in both gate $\left(\mathrm{S}_{\mathrm{IG}}\right)$ and drain $\left(\mathrm{S}_{\mathrm{ID}}\right)$ electrodes is obtained by calculating the Fourier transform of the autocorrelation functions. ${ }^{19,21,22}$ The autocorrelation functions are calculated from the instantaneous values of the current at the terminals obtained by means of the Ramo-Shockley theorem.

\section{RESULTS}

\section{A. Static characteristics}

Firstly, in order to present the influence of the tunnel injection on the static performance of the device, (a) $I_{G}-V_{G S}$, (b) $I_{D^{-}}-V_{D S}$, and (c) $I_{G^{-}}-V_{D S}$ characteristics are shown in Fig. 4 when considering $q \phi_{B n}=0.3 \mathrm{eV}$ and $A^{*}=10 \mathrm{~A} \cdot \mathrm{cm}^{-2} \cdot \mathrm{K}^{-2}$ in the HEMT of Fig. 1. The gate current, due to tunnel effect, severely depends on $\mathrm{V}_{\mathrm{GS}}$, since $T_{C}$ is markedly related to the potential profile in the region near the Schottky barrier. Thus, for higher absolute values of $V_{G S}\left(V_{G S}\right.$ more negative), this is, when approaching to the pinch-off region, the tunnel injection is stronger because of the smaller width of the potential barrier, which leads to a higher $T_{C}$. In the pinch-off region $\left(V_{G S}<-1.1 \mathrm{~V}\right)$ the influence of $\mathrm{V}_{\mathrm{DS}}$ is remarkable, also due to the modification introduced in the potential barrier. The (a) $I_{D^{-}} V_{G S}$ and (b) $I_{S^{-}} V_{G S}$ curves are plotted in Fig. 5 for several 

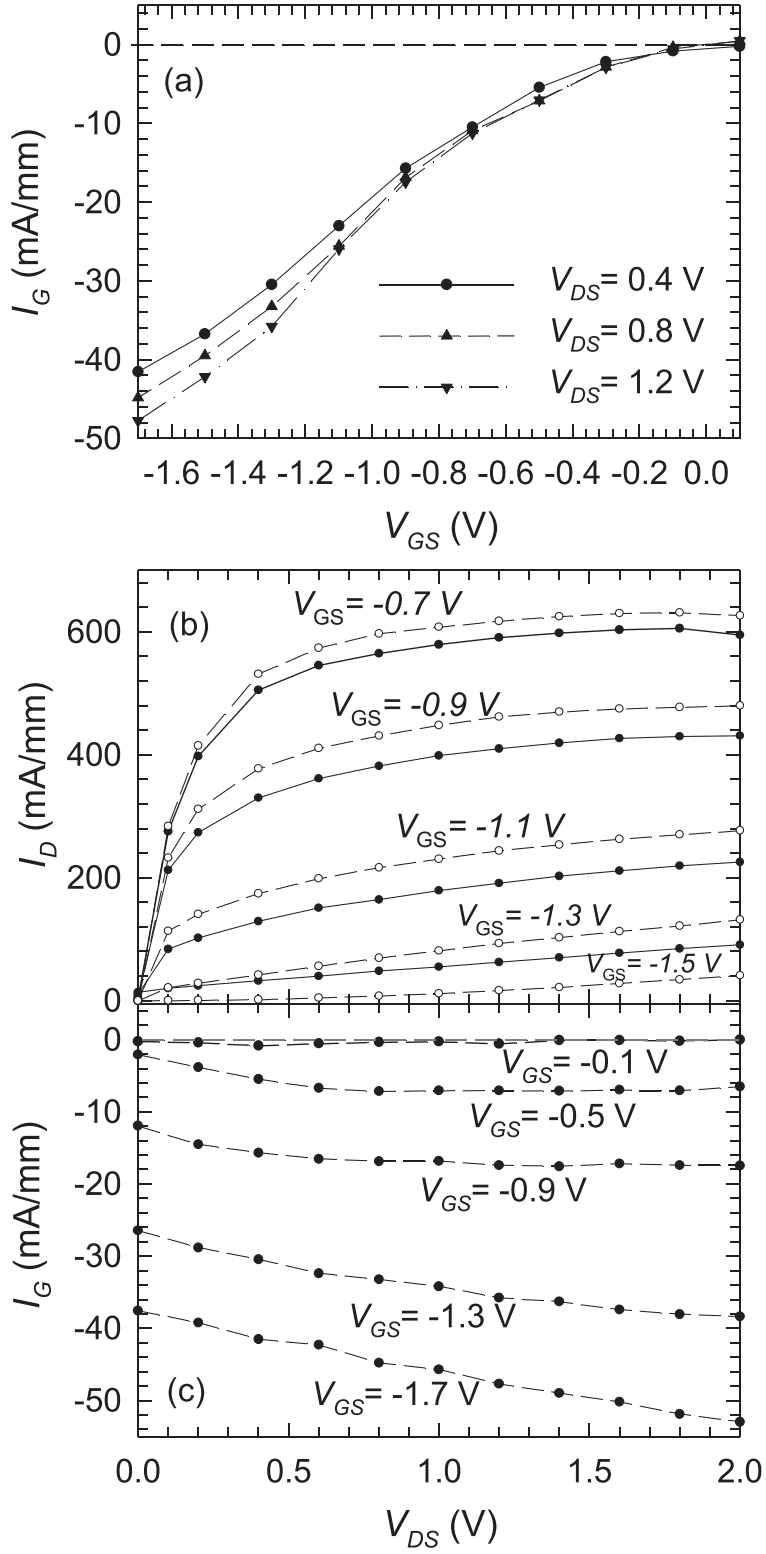

FIG. 4. (a) $I_{G}-V_{G S}$, (b) $I_{D}-V_{D S}$ and (c) $I_{G}-V_{D S}$ characteristics when considering tunnel effect being $q \phi_{B n}=0.3 \mathrm{eV}$ and $\mathrm{A}^{*}=10 \mathrm{Acm}^{-2} \mathrm{~K}^{-2}$.

values of $V_{G S}$, with and without considering tunnel effect in the simulations. Both cases are similar for the highest values of $V_{G S}\left(V_{G S}>-0.3 \mathrm{~V}\right)$ because the tunnel current is negligible. When $V_{G S}$ decreases from $V_{G S}=-0.3 \mathrm{~V}$ to $-1.3 \mathrm{~V}$, gate tunneling leads to a reduction of $I_{D}$ and thus seems to increase the threshold voltage of the device; this result being similar to that found in Ref. 25. Moreover, the experimental results in Refs. 26 and 27 confirm that a high gate leakage current is linked to a lower $I_{D}$. However, for $V_{G S} \leq-1.3 \mathrm{~V}$, the pinchoff is degraded, and non-zero values of $I_{D}$ and $I_{S}$ (which even takes positive values, produced by outgoing electrons) are obtained when tunnel effect is considered. The remarkable pinch-off degradation in the simulated device is consequence of the low value adopted for the barrier height. By considering a higher barrier height and, consequently, lower gate tunneling, the $I_{D}$ decrease would be softer and the pinch-off degradation, in case of being present, would appear at more negative $V_{G S}$ values.

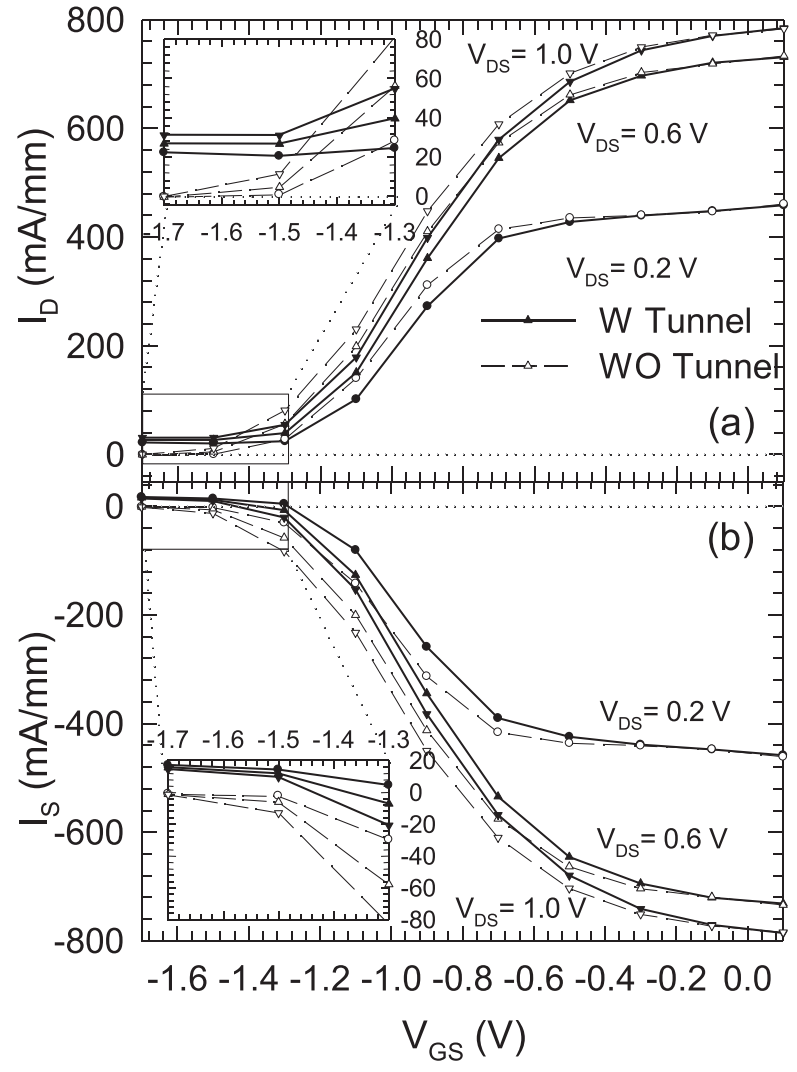

FIG. 5. (a) Drain and (b) source I-V characteristics with (solid lines) and without (dashed lines) considering tunnel effect in the simulations for $q \phi_{B n}=0.3 \mathrm{eV}$.

The influence of the tunnel injection of carriers in the $I-V$ characteristics can be explained in terms of the internal quantities of the device. Fig. 6 presents the potential profile along the channel section next to the heterojunction, with and without considering tunnel injection in the simulations, for $V_{D S}=1.2 \mathrm{~V}$ and two different values of $V_{G S}$. As well, the concentration (a) at the top of the channel and (b) along a cross section at the middle of the gate contact is plotted in Fig. 7 for the same bias conditions. As observed in Fig. 5, in open channel conditions, the values achieved for $I_{D}$ and $I_{S}$ are lower when considering tunnel injection. This happens

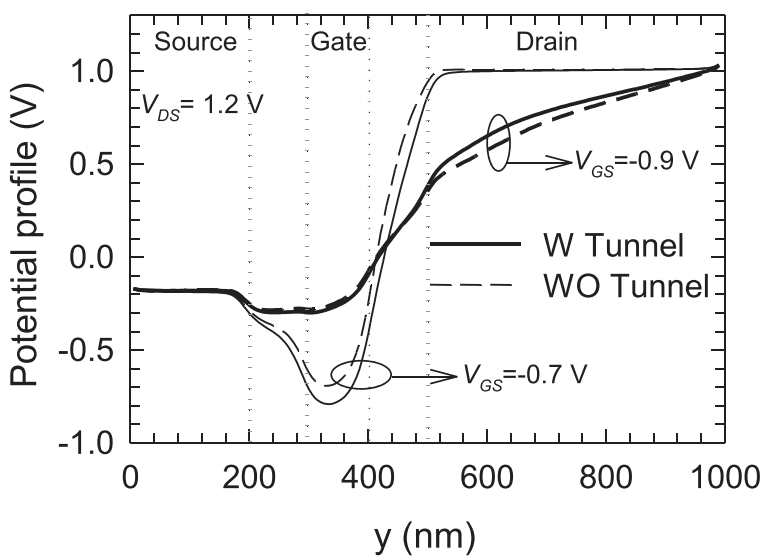

FIG. 6. Potential profile along the channel with $\left(q \phi_{B n}=0.3 \mathrm{eV}\right)$ and without considering tunnel effect for $V_{D S}=1.2 \mathrm{~V}$ and several $V_{G S}$. HEMT regions have been marked for comprehensibility. 

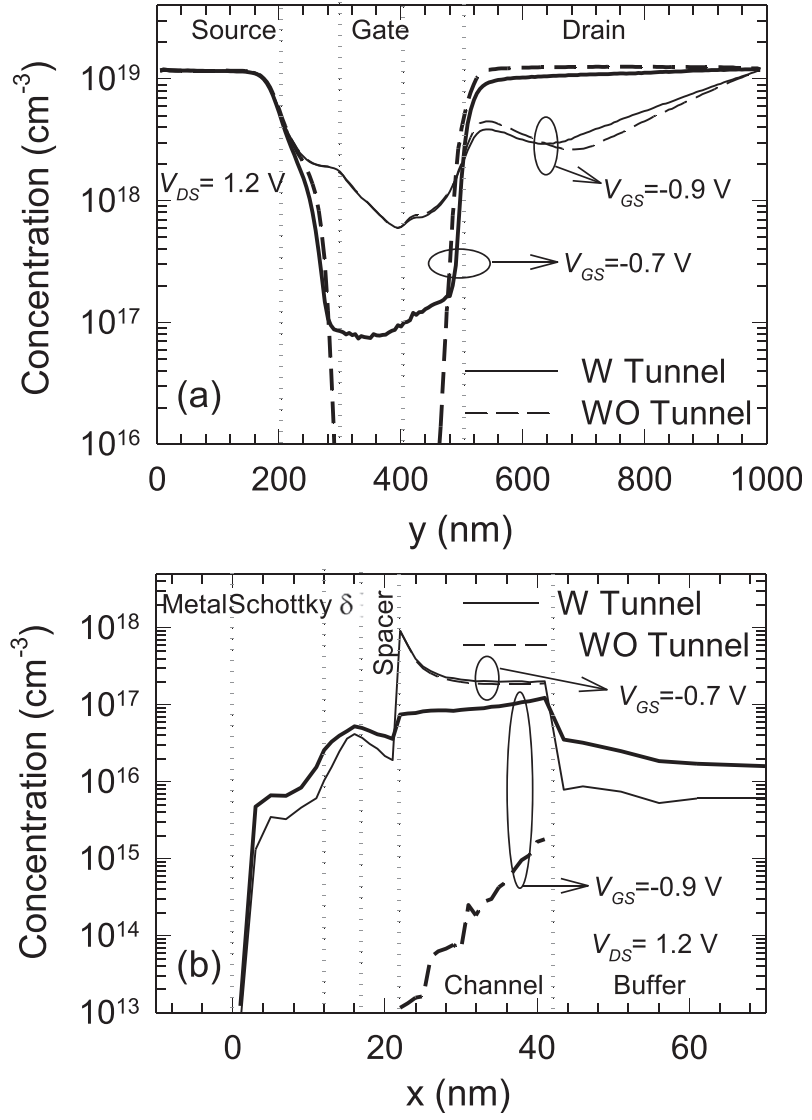

FIG. 7. Electron concentration (a) along the channel in the section next to the spacer, and (b) along the cross section in the middle of the gate contact, with $\left(q \phi_{B n}=0.3 \mathrm{eV}\right)$ and without considering tunnel effect, for $V_{D S}=1.2 \mathrm{~V}$ and several $V_{G S}$. HEMT regions have been marked for comprehensibility.

because of an increase in the potential barrier (Fig. 6) that controls the electron transport through the channel between source and drain electrodes. This increase is produced by the electrons injected by tunneling through the Schottky barrier at the gate electrode into the Schottky layer of the device. The amount of injected electrons increases when decreasing $V_{G S}$, and then $I_{D}$ and $I_{S}$ apparently head to an early pinch-off. However, for $V_{G S}$ negative enough to pinch off the channel (Fig. 6, $\mathrm{V}_{\mathrm{GS}}=-1.7 \mathrm{~V}, I_{D}=0$ ) in the absence of tunnel effect, when considering tunnel injection there is an extra injected charge from the Schottky layer into the spacer and, more significantly, in the channel (Fig. 7). In these conditions, even if the potential barrier is higher than in the absence of tunnel injection (Fig. 6), the pinch-off situation is avoided due to the presence of tunnel-injected electrons. This gate leakage current flows from the gate-region of the channel (and through the low-mobility layers) towards the source and the drain electrodes, with the consequent increase of both $I_{D}$ and $I_{S}$. The absence of a clear pinch-off situation is the most important drawback related to the presence of tunneling current.

Our model also allows us to check the influence of the IC effect on the global behavior of the device. In Fig. 8, the increment linked to the IC of (a) $I_{G}$ and (b) $I_{D}$ is presented as a function of $V_{G S}$. As expected, the consideration of IC enhances the injected tunnel charge into the Schottky layer,

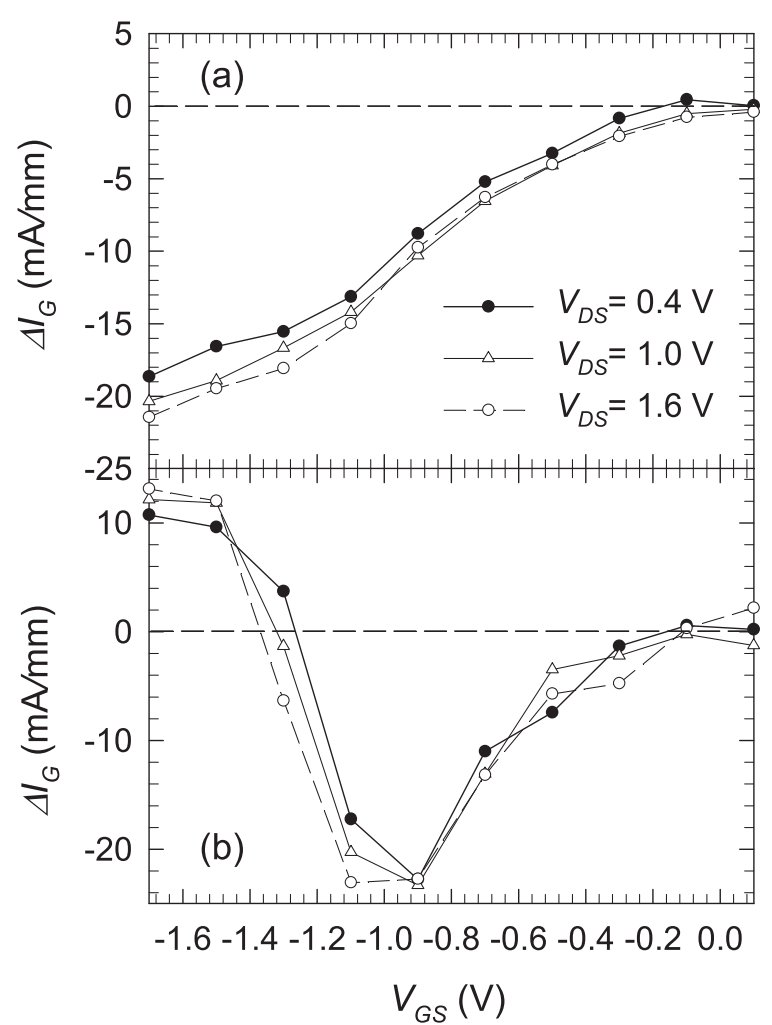

FIG. 8. (a) $\Delta I_{G}-V_{G S}$ and (b) $\Delta I_{D^{-}} V_{G S}$ characteristics when tunnel injection in the simulations is considered with and without including the Schottky (image charge) effect, for several $V_{D S} . q \phi_{B n}=0.3 \mathrm{eV}$.

which leads to an important increase in $\mathrm{I}_{\mathrm{G}}$, even reaching $40 \%$ of the value obtained when switching-off the IC effect in the simulations (for example, $\Delta I_{G} \cong 20 \mathrm{~mA} / \mathrm{mm}$ for $V_{G S}=-1.7 \mathrm{~V}$ while $I_{G} \cong 45 \mathrm{~mA} / \mathrm{mm}$ in the absence of IC effect). Regarding the influence of the IC effect on the drain current [Fig. 8(b)], the apparent premature pinch-off noticed for low values of $\left|V_{G S}\right|$ is strongly enhanced when this effect is considered in the simulation. However, when $V_{G S}$ approaches the pinch-off range, the opposite effect (increase of $I_{D}$ ) is enhanced by the extra current provided by the increase of tunnel injection.

\section{B. Noise behavior}

The noise study is carried out by analyzing the spectral density of current fluctuations in both gate and drain electrodes calculated as the Fourier transform of the corresponding autocorrelation functions. In Fig. 9, (a) $S_{I G}$ and (b) $S_{I D}$ are presented as a function of frequency, with and without considering the tunnel injection in the device, for several biasing conditions. The high-frequency peak due to plasma oscillations is noticed, as usual, ${ }^{28,29}$ in all cases. $S_{I D}$ presents an increase of the low-frequency plateau (beyond 1/f contribution) when tunnel effect is included in the simulations. Interestingly, there is an increase in $S_{I D}$ due to the gate tunnel injection, while this effect leads to a reduction in $I_{D}$ in open channel conditions. This increase in the drain noise is remarkable even when the tunnel effect is hardly noticeable in the $I-V$ characteristics, fact that must be taken into account when designing ultra-scaled HEMTs. Indeed, at $V_{G S}=-0.5 \mathrm{~V}$ when 


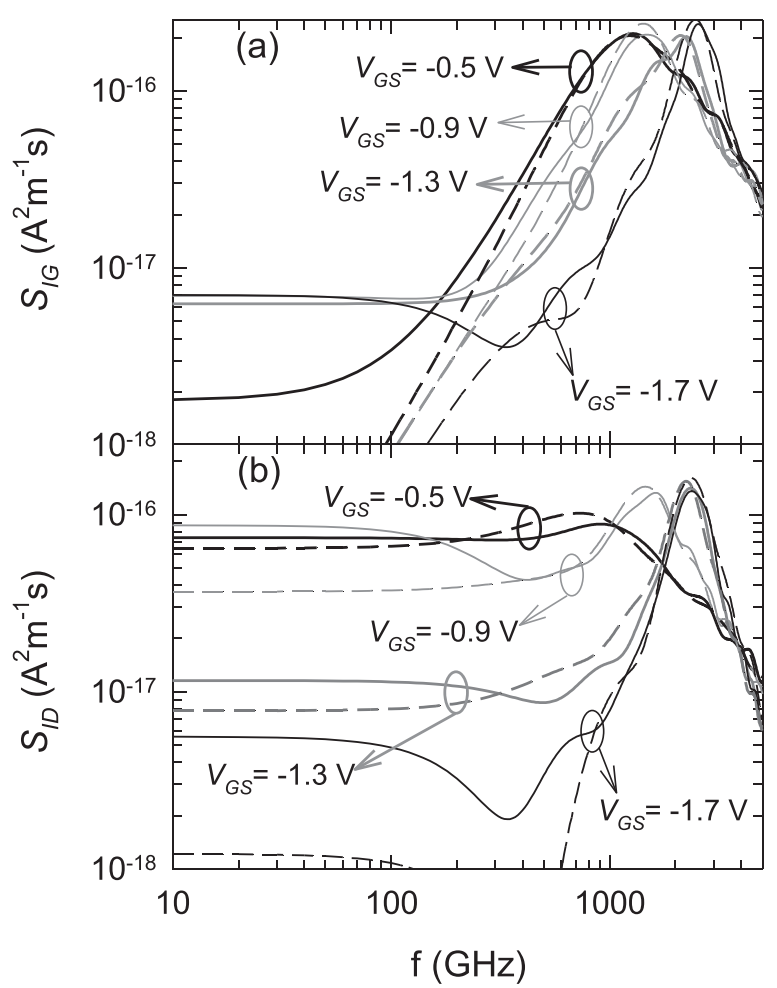

FIG. 9. (a) $S_{I G}$ (f) and (b) $S_{I G}$ (f) with $\left(q \phi_{B n}=0.3 \mathrm{eV}\right.$, solid lines) and without (dashed lines) considering tunnel effect in the simulations. $V_{D S}=1.0 \mathrm{~V}$.

the influence of tunneling is hardly noticeable in $I_{D}$, an increase of $S_{I D}$ is already observed. The origin of these effects is attributed to the fluctuations induced in the potential barrier controlling the current flow through the channel due to the charge injection into the Schottky layer. ${ }^{29}$ These potential fluctuations are strongly coupled with the drain current fluctuations, leading to the large increase observed in $S_{I D}$. This effect is of great interest when designing ultra-scaled HEMTs for low-noise applications, since gate tunneling can highly degrade the noise behavior without being clearly discerned in the static behavior.

As concerns $S_{I G}$, due to the appearance of a DC $I_{G}$ originated by the tunnel injection through the Schottky contact, a low-frequency enhancement in $S_{I G}$ takes place. In fact, since electrons are injected randomly into the device, full shot noise is expected to appear in the gate current, this being the

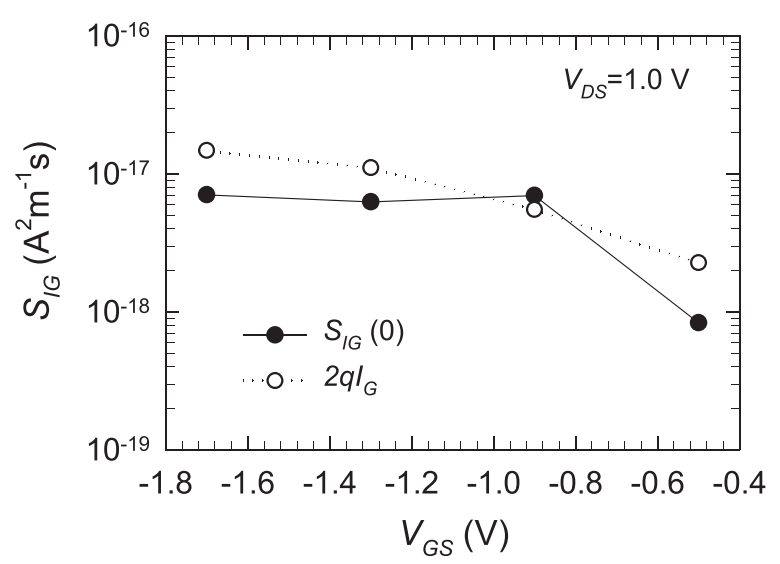

FIG. 10. $S_{I G}(0)$ and $2 q I_{G}$ for several biasing conditions. $q \phi_{B n}=0.3 \mathrm{eV}$. source of noise commonly associated to the gate leakage current, ${ }^{19,30}$ and confirmed experimentally in different FET gate structures. $^{31,32}$ To further illustrate this point, Fig. 10 presents the low-frequency values of $S_{I G}(0)$, calculated by using the instantaneous gate current values obtained by means of the Ramo-Shockley theorem, as compared with the theoretical value of full shot noise, $2 q I_{G}$. The comparison confirms the expectations.

\section{CONCLUSION}

By using the WKB model, the tunnel injection through the Schottky barrier at the gate electrode of InAlAs/InGaAs HEMTs has been analyzed by means of a 2D-MC simulator self-consistently coupled with a Poisson solver. Regarding the static characteristics, we have confirmed that the tunnelinjected carriers into the Schottky layer of the HEMT lead to an increase in the potential barrier that controls the electron flowing through the channel. As a consequence, lower values of $I_{D}$ are obtained, with an apparent premature pinch-off for relatively high values of $V_{G S}$. However, when $V_{G S}$ is negative enough to approach the pinch-off conditions, non-zero drain and source currents are observed as a result of the gate leakage current. In fact, the absence of a pinch-off behavior is one of the more important problems regarding the static behavior of the device due to the appearance of strong tunnel effect; this is, when the devices are extremely scaled down.

With respect to the noise behavior, a low-frequency (beyond 1/f) current-noise increase has been observed in both gate and drain electrodes. On the one hand, the gate currentnoise has been identified as shot noise due to tunnel injection. On the other hand, the enhanced low-frequency plateau found in $S_{I D}$ has been attributed to the fluctuations in the potential barrier (controlling the carrier transport through the device) caused by the tunnel-injected electrons. This noise increase is very significant even if the tunnel effect is not noticeable in the I-V characteristics, which must be taken into account when designing scaled HEMTs for low-noise applications.

\section{ACKNOWLEDGMENTS}

This project has been supported by the Consejería de Educación, Junta de Castilla y León through Project SA052U13.

${ }^{1}$ R. Lai, X. B. Mei, W. R. Deal, W. Yoshida, Y. M. Kim, P. H. Liu, J. Lee, J. Uyeda, V. Radisic, M. Lange, T. Gaier, L. Samoska, and A. Fung, Tech. Dig. - Int. Electron Devices Meet. 2007, 609.

${ }^{2}$ K. Shinohara, Y. Yamashita, A. Endoh, I. Watanabe, K. Hikosaka, T. Mimura, S. Hiyamizu, and T. Matsui, in Proc. 16th Int. Conf. Indium Phosphide and Related Materials, IEEE Catalog 04CH37589, Kagoshima, Japan (2004), p. 721.

${ }^{3}$ Y. Yamashita, A. Endoh, K. Shinoara, K. Hikosaka, T. Matsui, S. Hiyamizu, and T. Mimura, IEEE Electron Device Lett. 23, 573 (2002).

${ }^{4}$ J. Mateos, T. González, D. Pardo, S. Bollaert, T. Parenty, and A. Cappy, IEEE Trans. Electron Devices 51, 1228 (2004).

${ }^{5}$ J. Mateos, T. González, D. Pardo, V. Hoel, and A. Cappy, IEEE Trans. Electron Devices 47, 1950 (2000).

${ }^{6}$ J. Mateos, T. González, D. Pardo, V. Hoël, H. Happy, and A. Cappy, IEEE Trans. Electron Devices 47, 250 (2000).

${ }^{7}$ B. H. Bransden and C. J. Joachain, Introduction to Quantum Mechanics (Longman Scientific \& Technical, New York, 1987).

${ }^{8}$ R. Rengel, E. Pascual, and M. J. Martín, IEEE Trans. Electron Devices 28, 171 (2007). 
${ }^{9}$ B. G. Vasallo, N. Wichmann, S. Bollaert, Y. Roelens, A. Cappy, T. González, D. Pardo, and M. Mateos, IEEE Trans. Electron Devices 54, 2815 (2007).

${ }^{10}$ B. G. Vasallo, J. Mateos, D. Pardo, and T. González, J. Appl. Phys. 94, 4096 (2003).

${ }^{11}$ W. Shockley, J. Appl. Phys. 9, 635 (1938).

${ }^{12}$ S. Ramo, Proc. IRE 27, 584 (1939).

${ }^{13}$ H. Kim, H. S. Min, T. W. Tang, and Y. J. Park, Solid-State Electron. 34, 1251 (1991).

${ }^{14}$ E. Pascual, R. Rengel, and M. J. Martín, Semicond. Sci. Technol. 22, 1003 (2007).

${ }^{15}$ E. Pascual, R. Rengel, N. Reckinger, X. Tang, V. Bayot, E. Dubois, and M. J. Martín, Phys. Status Solidi C 5, 119 (2008).

${ }^{16}$ E. Pascual, M. J. Martín, R. Rengel, L. Larrieru, and E. Dubois, Semicond. Sci. Technol. 24, 025022 (2009).

${ }^{17}$ L. Sun, X. Y. Liu, M. Liu, G. Du, and R. Han, Semicond. Sci. Technol. 18, $576(2003)$.

${ }^{18}$ C. K. Huang, W. E. Zhang, and C. H. Yang, IEEE Trans. Electron Devices 45, 2079 (1998).

${ }^{19}$ T. González, J. Mateos, D. Pardo, O. M. Bulashenko, and L. Reggiani, Semicond. Sci. Technol. 13, 714 (1998).
${ }^{20}$ C. R. Crowell and S. M. SZE, Solid-State Electron. 9, 1035 (1966).

${ }^{21}$ A. Cappy, IEEE Trans. Microwave Theory Tech. 36, 1 (1988).

${ }^{22}$ T. Kuhn, L. Reggiani, and L. Varani, Phys. Rev. B 42, 11133 (1990).

${ }^{23}$ D. A. Neamen, Semiconductor Physics and Devices (Irwin, Boston, 1992).

${ }^{24}$ L. Racko, J. Pecháček, M. Mikoláček, P. Benko, A. Grmanová, L. Harmatha, and J. Breza, Radioeng. J. 22, 213 (2013).

${ }^{25}$ K. Kalna and A. Asenov, Solid-State Electron 48, 1223 (2004).

${ }^{26}$ S. Karboyan, J. G. Tartarin, N. Labat, and B. Lambert, in IEEE Proc. 22nd International Conference on Noise and Fluctuations (ICNF) (2013).

${ }^{27}$ P. Lei, P. Yan, P. Winju, W. Liang, L. Chengzahn, L. Jian, Z. Yingkui, and W. Ke, J. Semicond. 30, 054001 (2009).

${ }^{28}$ B. G. Vasallo, J. Mateos, D. Pardo, and T. González, J. Appl. Phys. 95, 8271 (2004).

${ }^{29}$ B. G. Vasallo, H. Rodilla, T. González, G. Moschetti, J. Grahn, and J. Mateos, Semicond. Sci. Technol. 27, 1003 (2012).

${ }^{30}$ F. Danneville, G. Dambrine, H. Happy, P. Tadyszak, and A. Cappy, SolidState Electron. 38, 1081 (1995).

${ }^{31}$ M. Manghisoni, L. Gaioni, L. Ratti, V. Re, V. Speziali, and G. Traversi, in IEEE Nuclear Science Symposium Conference Record (2007), p. 2503.

${ }^{32}$ C. Ciofi, F. Crupi, C. Pace, and G. Scandurra, IEEE Trans. Instrum. Meas. 52, $1533(2003)$ 